\title{
Notes from the podium of an Online Orchestra
}

\author{
Jonathan J. Hargreaves
}

\section{Abstract}

Online Orchestra placed significant demands on its conductor, with a role that included rehearsing and directing remote musicians over the Internet. This article presents a firsthand reflective account from the conductor of Online Orchestra, including details of trials, rehearsals and the final performance. Practical considerations such as conducting technique, visual framing and ensemble seating are considered, as are reflections on conducting in a latency-rich environment. This leads to the conclusion that many traditional approaches to conducting apply in telematic performance, and the suggestion that there is significant scope for musicians to learn and grow in ability when making music online.

Keywords

Online Orchestra; telematic performance; conducting; rehearsing; latency; eye contact 
Telematic performance has vast potential to enable music that has previously been impossible. On the Internet, musicians can simply disappear or pop up out of nowhere,ग in a way that they cannot in traditional, offline performance; or sounds might be re-routed so as to appear in one node but not in others, for example. This offers whole new territories in which and with which composers can work. However, the history of telematic performance is somewhat dominated by improvisation (see Rofe et al. 2017a), and this implies a more egalitarian ensemble than the traditionally hierarchical 'orchestra'. In essence, improvised music is an ongoing re-negotiation of the roles played by the musicians, and this is far more akin to the quintessentially distributed quality of networks than orchestral music, in which instrumental sections, under their leaders, play prescribed roles in relation to one another, all under the direction of a conductor, who interprets a composer's score. At the outset of the project, these background issues posed questions to the technologists, composers and performers of Online Orchestra, and the collaboration between these groups has been crucial in achieving online orchestral performance.

\section{Early trials}

In the early stages, as we explored various network structures and their implications for composition and performance, we varied approaches to conducting, exploring a range of hand signals that might work well in an online environment. As shown in Figure 1, trials were undertaken with four musicians, located in different rooms that were connected over the network. Improvisatory 'conduction' proved to be particularly effective from the 
point of view of user experience: conduction is 'a way of organizing musical interaction by means of a set of codified gestural cues directed by the conductor to instrumentalists'

(Veronesi 2014: 468). This group improvisation technique grants to musicians the autonomy to interpret the gestures of the conductor at the level of $\mathrm{pitch} / \mathrm{attack} / \mathrm{sound} /$ dynamic, and this gave the players a sense of interconnection and playfulness that they reported enjoying. It also enabled the absorption of network latency within the improvisation.

Figure 1: Early conducting trials.

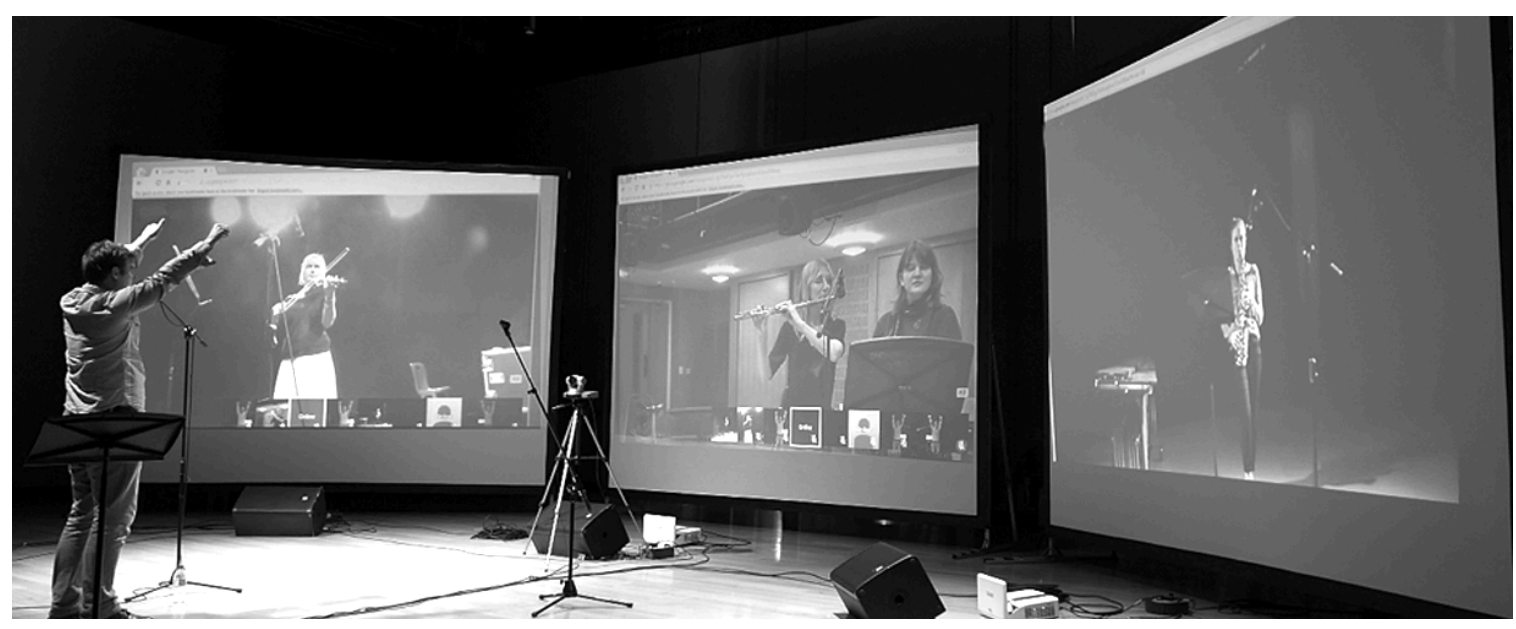

However, one of the aims of Online Orchestra was to enable a large-scale ensemble to play notated, pulsed music over the network (see Rofe et al. 2017b). Moreover, there was a need for the musical activity in the network to be familiar to the orchestral members, given our focus on amateur and community music-making. Retrogressive though it may seem given the potential offered by telematic performance, the aim was to enable conventional orchestral music. Taking this model provided focus for the project, and hopefully later Online Orchestras will benefit from the establishment 
of this convention, which will - it is to be hoped - enable subsequent deviation and innovations.

Paradoxically, the very technology that enables connection places restrictions on interaction. Leaving aside the implications of latency, there are inevitable limits on the extent to which, and the domains in which, musicians can interact in this environment. Eye contact between individuals in different nodes is impossible, for example; that the audio was reduced to a mono signal per node, and the $3 \mathrm{D}$ conductor was reduced to a $2 \mathrm{D}$ video feed, significantly limits the spatial information available to musicians, in turn reducing the scope to play with how sounds mix, dynamically, and how physical gestures relate to each other, and to sounding results. Such channels of communication are central to pushing music-making to higher levels; there is practice-based research to be done with and by professional musicians as the Online Orchestra system develops.

\section{Conducting Online Orchestra: Outward visual appearance}

In telematic performance, as in conventional orchestral performance, at a fundamental level, the conductor's role is to embody the unity of the sound, giving visual information regarding timing, phrasing and expression. Similarly, in online rehearsal, the purpose is to facilitate ensemble playing and to exploit the relationships inherent in the score in such a way that the players in the sections (nodes) perceive musical interaction with and across the whole ensemble (network). Rehearsals and performances that we have carried out so far using Online Orchestra technology suggest that with the present system, appropriate repertoire (see Rofe and Geelhoed 2017), and players of an appropriate level and 
experience (discussed below), there is scope for improving ensemble playing, including working on the nuance of the composite sound: there is a role for the conductor. As more composers and performers use this technology, the issues and obstacles to musical interaction that arise may well lead to new solutions; certainly any notional 'online conducting technique' is in an early developmental stage and may evolve into something very different.

In its outward appearance, the method of physical gesture used in Online Orchestra was the same as conventional 'in the room' conducting. There is little empirical research on conducting technique (examples include Byo 1990; Byo and Austin 1994; Lee et al. 2005), perhaps because conducting itself is so highly idiosyncratic. However, there are two generally accepted approaches to beating patterns (the trajectory of the baton through the beats of the bar). Some conductors beat such that the exact point (ictus) of every beat in the bar is located at the same point in space, such that the arm movement between the beats delineates the 'geography' of the bar. In Online Orchestra, however, the players play to a flat image on a screen rather than to a $3 \mathrm{D}$ person, meaning that the beating pattern described above is, therefore, inappropriate. Rather, there is a special need to make sure that the locus of each beat of the bar is at a different and consistent place from one bar to the next. (Diagrams of the beating patterns used can be found in Max Rudolf's The Grammar of Conducting (Rudolf 1995).) The appearance of the upbeat and the downbeats are naturally distinct: players see the outside, upper side of the hand at the bottom of the downbeat and (albeit that the fingers grasp the baton handle covering the palm) the inside of the hand at the top of the upbeat. To make sure the beat is as clear as possible, it is important to make a pronounced lateral movement between 
the middle beats of a given bar. In making the horizontal movement between beats two and three of a $4 / 4$ bar, for example, it is important for the conductor to place beat three distinctly to the right of the locus of beat four. This applies to conducting technique in general, but it is especially important in online conducting. Indeed, musicians commented on this issue when playing to the screen in the testing stages, whereas arguably many an amateur conductor has escaped with a less precise beat three in conventional performance without musicians mentioning it.

\section{It's all about the shirt!': The televisual image}

My tongue-in-cheek insistence at the start of the project that my choice of shirt would come to be one of the most crucial links in the communication chain became a running joke over the course of the project. However, in the testing phases, musicians commented upon the need for the right sort of attire for conducting (luckily I had brought a few options). It is advisable for any conductor to wear dark/black clothes in performance and rehearsal - to maximize contrast with the (usually white) baton. Online, the issue is more pronounced as that contrast is captured and transmitted in the televisual image; in the final rehearsals, we established that rolled-down shirt sleeves made a significant difference to the ease with which remote musicians could follow the beat. Failing dark clothes, non-patterned, block colours are advisable. The cable for the headphones should run down the back, rather than across the front of the body, where it might get in the way of the arms. 
The significance of clothing is a part of the broader issue of setting the screenshot, and the same principle applies to other parameters: the ideal is to conduct against a black background, for instance. Again, if a black backdrop is not available, the conducting should be in front of a non-patterned, monochrome flat surface; in one trial, I was based in a room in front of bookshelves, and musicians reported the need to cover these in order to reduce visual noise around the baton. Likewise, the music stand was set at waist height or just above, as musicians need to see the top half of the conductor's body clearly. Good lighting was also crucial, in particular avoiding any backlighting that might render the conductor into shadow.

\section{Conducting Online Orchestra: Inner perceptual experience}

The conductor's inner experience in Online Orchestra is markedly different from conventional conducting. The principal difference is that the system's latency control forces the sound of the musicians - or, depending on the structure of the network, part of the sound - to arrive back to the conductor two beats after the corresponding cue has been given (see Rofe and Reuben 2017). This poses a perceptual challenge to the conductor, although in actual fact it is relatively easy to acclimatize. A useful analogy is that of an organist playing in a church with the console on one side of the quire and the pipes on the other: as long as the delay is constant, one can track the continuum of the sound. In truth, it is not a perfect analogy: arriving precisely two beats of tempo late, Online Orchestra's signal delay is of a different order. Nonetheless, the principle is the same (and, like so many notes here, applies in conventional performance): it is crucial to 
know the score well enough to be confident of where you are up to and well enough for muscle memory to kick in if there are moments of uncertainty in performance.

It is far easier to adjust to the latency where there is an isolated conductor node, which is to say where there are no musicians in the same node as the conductor. In such a case - as in Online Orchestra's pilot performance - the challenge posed to perception is relatively simple: all of the sound is two beats late. We trialled, though ultimately rejected, a scenario in which the conductor node was shared with musicians (in this case, a violinist and a violist were in the room with the conductor). Thus, those two musicians performed with no latency from the conductor's perspective, whereas there was a twobeat delay on sound from the remote musicians (in this case, a string quartet in a second node). This is a rather different and far greater challenge, as the conductor must learn the music with that relationship in mind. On that occasion, marking up the score appropriately was crucial, with cues pencilled in two beats ahead of the printed parts; the task was to conduct the pencil quartet cues and the ink violin/viola cues, but in rehearsing the piece to listen to the sound of the printed score - a difficult cognitive split, but again, one that can be overcome. In fact, for this trial, the composer had been asked to prepare two scores, one showing the resultant sound in the string quartet node and the other showing that in the conductor node, the latter being the one marked up with the pencil cues.

It may seem dismissive simply to say that one can easily acclimatize to the latency-rich context of the Online Orchestra network; it is difficult to describe a learning process that is predominantly procedural rather than declarative. An implication of the latency is that there is an emphasis on conducting continua, rather than events. For 
instance, it is, on occasion, a useful rehearsal technique to ask ensembles to play single impulses - for example as loud and short, or as quiet and short, a chord as possible - to focus on playing together, and to illustrate, establish and hopefully to extend, the expressive extremes of various aspects of the group's overall sound. To isolate a single event like this in the Online Orchestra network highlights the dislocation of gesture and sound. In the nodes, the visual appearance is fine: the ensemble plays on the beat with the conductor (albeit the sound of the other nodes arrives one beat later); for the conductor, the sound arrives after the single cue has been given. This is not a practical problem per se, but it is worthwhile for musicians to recognize that the essence of what they are working with is a superimposition of continua (the audio/video data streams), rather than a sequence of events. Accordingly, it may be advisable to learn and think of the music to be played in ways that emphasize continuity.

In rehearsals and performances, we used a click track in the conductor's headphones, and inevitably this produced a neater, tighter sound given that this metronome is precisely locked to the network latency. The audio set-up eliminates almost all of the echoic feedback loops although there are still occasional perceptible echoes in the signal (see Prior et al. 2017). This might suggest that it is actually harder to play out of time than it is to play in time with the metronome, though that is yet to be found out. Now that various procedural aspects of Online Orchestra are in place, it will be interesting to play without a click in a project with more rehearsal time than has been available so far. We may find at that point that the metronome plays an essential role in enabling continuity within online orchestral performance: to introduce scope for rubato 
may be, effectively, to allow what is perceived as a distributed error to spread through the network.

A consequence of Online Orchestra's approach to dealing with latency - one that relates to the temporal dislocation of gesture and sounding result - is that there sometimes arise in performance moments of direct mismatch between the two. Presuming that the conductor is based in an isolated node, he or she must lead the ensemble through crescendos, give accents, indicate dynamic changes, and so on, two beats before they are heard in the conductor node. This leads to situations in which, for example, the conductor gesticulates to create a fortissimo staccato accent, whilst hearing the ensemble playing pianissimo sustained chords, or vice versa. (For the musicians in the nodes, of course, there is no such mismatch.) Counterintuitive though this may be, it was easy enough to process. Situations like the one described above imply the use of a similar cognitive skill. Thus, provided one knows the score well enough to parse it in larger chunks, such moments do not obstruct conducting online. Indeed, as described above, the task knowing the score - is the same for online and 'offline' conducting.

One final issue to note here is that of changing time signatures and irregular metres, which cause slight disturbances in the constancy of the relationship between the conductor's beat and the (delayed) sound. Figure 2 shows a simple example of two 3/4 bars amid a span of $4 / 4$ bars, and how this manifests in what the conductor hears. In the 3/4 bars, the conductor gives an upbeat while hearing a downbeat, as is inherent to triple time with a two-beat delay. The point, however, given that one is conducting continua, is that the pattern changes to a different symmetry from the surrounding $4 / 4$ bars. The challenge created by situations like this is only slight, as beating patterns themselves are 
habitual, and do not require focused attention even for conductors of moderate experience. Nonetheless, Online Orchestra conductors should be aware of this.

Figure 2: Metrical changes in a latency-rich environment.

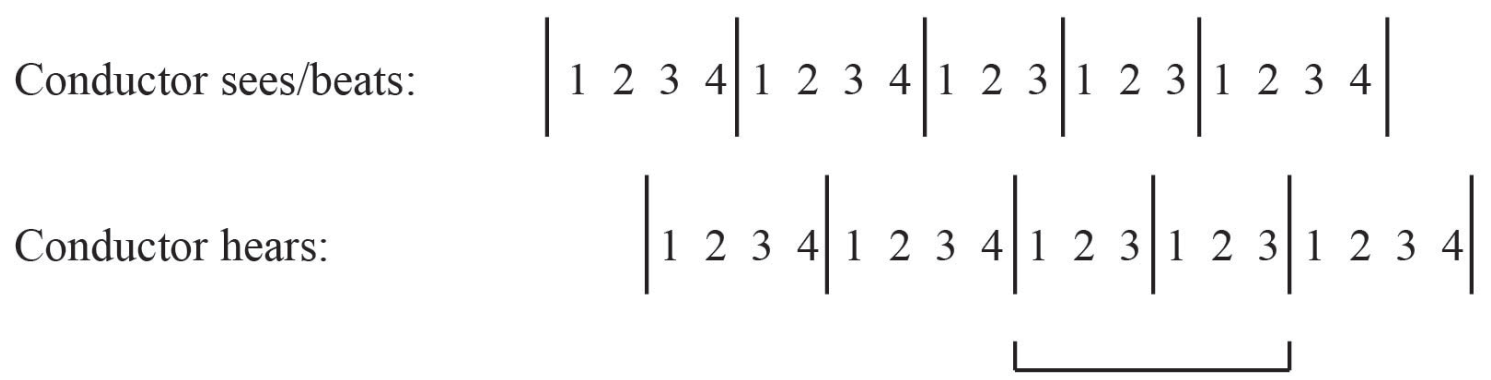

Asymmetrical time signatures present more of a challenge. In 7/8 time, for example, the grouping of the seven quavers within the bar $(2+2+3 ; 2+3+2 ; 3+2+2$ and so on) implies beats of different lengths, whereas the delay within the network remains constant (at a crotchet, or, in this context, two quavers). Further, music that uses irregular and asymmetrical time signatures often plays on rhythm and metre, implying continual regrouping within changing time signatures. This leads to situations such as the one shown in Figure 3, in which the asymmetry of the bar means that the conductor's beat does not correspond to the strong beats in the ensuing audio signal.

Figure 3: Asymmetrical metres in a latency-rich environment.

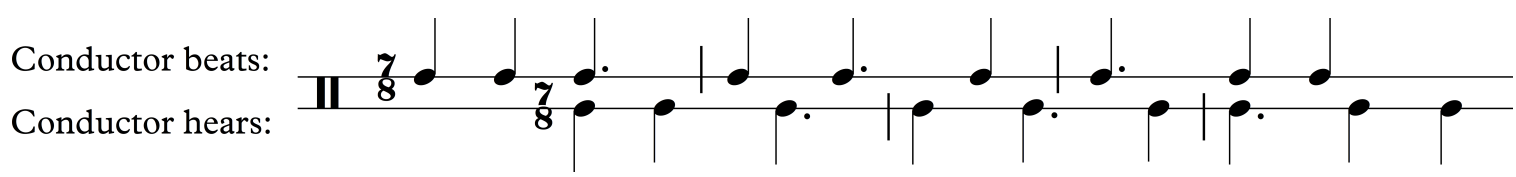

In addition, Figure 3 merely shows the structure of the (imagined) $7 / 8$ bars, rather than the orchestral texture, which could be far more rhythmically elaborate. Direct 
precedents for this kind of conductor-beating-against-the-ensemble situation abound in early modernist works such as Stravinsky's L'histoire du Soldat, a staple work for conducting competitions and master classes. There is an additional level of challenge here, however, particularly in rehearsal: as well as focusing attention on complex beating patterns, the conductor must at the same time listen to the highly syncopated sound. To perform these two mental tasks simultaneously is cognitively very demanding, although as with the other parts of the conductor's role it improves with practice.

\section{Contextual considerations for a virtual space}

The issues discussed above relate to coordinating of the 'virtual time' implied (even created) by Online Orchestra's latency control - while the conductor is two beats ahead of the ensemble, the sounds made in each of the nodes are one beat ahead of those in the other nodes, according to the score (see Rofe and Reuben 2017). Just as there is no definitive 'now' at any point on the network, there is also no definitive 'here'; that is, Online Orchestra musicians play in a 'virtual space', and this has implications for the conductor.

Approaching this issue from an abstract perspective and moving towards practical considerations, there is the issue of balancing the ensemble. Online Orchestra enabled the connecting of multiple acoustic spaces. Part of the conductor's job was to manifest those links and to find ways to enable the ensemble to embrace the acoustic space, just as in an unfamiliar concert venue. Various approaches were taken to this challenge, particularly in rehearsal. One ensemble exercise was to ask for the orchestra to play a sustained chord 
lasting two bars, with one node playing at a constant mezzo forte, and the others starting pianissimo, but making a gradual crescendo to the same dynamic as the louder node.

As testimony to the quality of the audio signal, perhaps the pinnacle in the musical achievement of Online Orchestra came in the first online tutti rehearsal. One of the commissioned pieces was built of melodic lines, treated in multiple canon to create chords whose colour changes as different instruments play the line - or, in effect, pass through the harmony. This places a particular emphasis on achieving an appropriate composite timbre, and, concomitantly, the importance of balance and intonation is heightened: any ensemble that is to blend and create a single, composite sound must be in tune. In rehearsal, the group was able in real time to listen for the sound of the leader of the first violins as a reference point for tuning, and so to focus the global sound. This was a truly pivotal moment, which proved beyond doubt that effective rehearsal can be achieved using Online Orchestra technology.

Another implication of the abstract 'virtual space' in which online performance occurs prompts two interrelated questions that require concrete answers: what is the seating layout for an online orchestra and, beyond that, how does one cue the players, given the lack of direct eye contact? There is no 'correct' seating plan; only those that are more or less advantageous. After some experimentation with eye lines between musician, music stand and the televisual image of the conductor, we adopted the solution of asking players to sit in single arcs around the screens, with the higher instrumental parts at the left extreme, moving down to the lower ones at the right end of the arc. The cues required by the repertoire were generally based either in a distinct register or were addressed at entire nodes. Thus, I was able to turn to the appropriate direction to bring particular 
instruments in. The exception to that system was the choir, for whose cues I looked directly into the camera, and mouthed the first few words of the ensuing musical phrase. The musicians were able to follow that direction, although this is an inevitable compromise given the lack of real eye contact.

\section{Virtual drawbacks and actual benefits}

Online Orchestra is based on conventional orchestral performance, and in relation to that model it is tribute to the achievements of the technologists on the project that meaningful musical interaction can occur. In rehearsal, we were able to work together on the detail of the scores, to clarify textures and to improve ensemble. There are undeniable shortcomings, however, which create obstacles to group interaction: albeit that conductors can quickly adapt to the two-beat delay, it is a tautology to point out that one is not as connected with the sound as in conventional orchestral music-making; as explained above, eye contact between individuals is a major part of music-making; the relaying of the conductor's image on a 2D screen rather restricts the range of his or her movements (although, arguably, in some ways this makes for better technique).

The benefits of the system clearly outweigh these drawbacks, however - indeed, these are drawbacks in relation to a nineteenth-century approach to music-making. Away from virtuality and its implications for performance, the large-scale ensemble musicmaking enabled by Online Orchestra offers opportunities for educational, musical, personal, social and emotional growth and development to its members and membercommunities. It is too early in the project to measure such benefits; given the 
developmental nature of the research so far, the emphasis has been on technological invention and exploration. Suffice to say that there is definite scope for carrying out quantitative and qualitative research on these aspects of Online Orchestra. For now, the discussion below offers speculations as to the many benefits of the project for its members and outlines some of the assumptions and motivations that drove particular decisions regarding its social and musical structure.

\section{Structuring the ensemble}

At the start of the project, the brief was wide open: an 'Online Orchestra' could encompass an ensemble made up of any instruments in any community in West Cornwall, and the idea was that our work would also enable other future Online Orchestras elsewhere, particularly in other remote communities. Our project partners the Cornwall Music Education Hub kindly provided insider knowledge as to the make-up of the musical communities whom we might approach. However, part of the remit was that the project ought to be repeatable in other, unknown communities, where there might be very different human and musical resources. Inevitably, the lack of restrictions was more of an obstacle to decision-making than it was an aid, leading to circular discussions: we had no definite means of knowing the make-up of the musical communities we were to approach on the one hand; on the other, we had the freedom to approach any community. At that stage, a guiding principle for many of the musical decisions regarding the makeup of Online Orchestra was that we ought not to impose the project on the communities we were approaching. Rather, the structure of the ensemble ought to be flexible enough 
to be embedded seamlessly in future participant communities - following our reasoning, theoretically this ought to strengthen the integration within those communities, and open up the potential development of their members.

Seeking to conceive an ensemble whose structure would resonate with that of any musical community, we chose to base Online Orchestra around a string orchestra, given that future iterations of the project would likely be run by county music services (with attached youth orchestras) or as part of outreach programmes for professional orchestras; and a higher voice choir, on the basis that singing is possible in all communities, and would likely be performed by school groups. Beyond this, the brief for the composers in the pilot performance was to write parts for one node consisting of a teacher with differentiated parts for pupils at various stages of development (this decision building in scope for 'duet rehearsals' in individual lessons); and to score the other node as SATB, initially, to be re-orchestrated later for the specific instruments in the ensemble. In addition, we agreed that we would seek players of ABRSM Grade 6 standard or above, feeling that this level of expertise was needed for the musicians to be able to concentrate on the ensemble playing (to watch the conductor) rather than to devote a disproportionate level of attention to individual technique. Only then did we approach community groups, and as explained below, this level of thinking seemed to pay off in the eventual ensemble.

\section{Benefits for the musicians}

Undeniably, the major benefit of Online Orchestra for its members is that it gives them access to large-scale ensemble performance, meaning that they need not travel miles to 
participate. Speaking with some of the young flautists on Isles of Scilly, they said that one of the things they had particularly enjoyed about music trips to the mainland was that they sometimes saw instruments to which they would not have access otherwise; a harp, for example. To be clear: the Scilly Isles have a remarkably strong and highly active musical community given their small population - indeed, as part of Bournemouth Symphony Orchestra's intervention there, various large ensembles have started up on the islands since our project. Nonetheless, the access to orchestral performance that Online Orchestra was able to provide to Five Islands' School on St Mary's is iconic of the potential benefits of telematic performance.

Generally speaking, the music-educational benefits of Online Orchestra for the individual members were the same as those for similar conventional music education projects. Some of the rehearsals for the flutes in the Isles of Scilly, for example, took the form of rhythm workshops in response to one of the pieces. Another example in which musical skills were enhanced by the online environment, reported by teachers within the string node, was that their pupils were learning to follow a conductor - perhaps the $2 \mathrm{D}$ appearance of the conductor on-screen might explain the emphasis on this skill in performance.

Examples of how the technology enabled us to work on and improve the overall ensemble sound are given above. An important issue to address, regarding the benefits of online performance, is what might be called its 'musico-social' aspects. Despite earlier plans to approach a pre-established group, the string orchestra for the premiere comprised children aged 11 and up, local amateurs, professional musicians working in the area of Truro, Cornwall, and also members of the Philharmonia Orchestra, who kindly joined us 
for the concert. It was notable in rehearsals that these different types and levels of experience created a group in which there were multiple levels of support. Young pupils sat alongside their teachers, or other adults, playing the same music, and this made for a collaborative and cooperative atmosphere within the group.

This is not specific to online performance, however - the social structure of the string orchestra was established before the rehearsals extended to go online. At that point, however, perhaps one of the advantages of online performance did start to become apparent: the musicians in each node were, at that point in the project, representing their community to other ones. Thus, various features of the pieces - such as moments when an individual musician leads the entry of all musicians in that node - became moments in which those musicians might take added pride; for the 'tempo ad lib.' repeated figures in one of the pieces, the section leaders were followed far more faithfully. In short, as we had hoped, the connection outwards to other communities (telematic performance) seemed to strengthen the inner ties of each group.

\section{Conclusions}

The potential opened up by telematic performance is vast, and the Online Orchestra system offers scope for a great deal of research. A particularly exciting avenue of research will be to see what professional musicians can achieve on the network, both improvisers and those playing notated music. However, increasing the level of musicianship that is possible on the network is only one of many goals - and indeed, it is 
actually a secondary goal. Accessibility lies at the core of the Online Orchestra project, since that is surely the overriding benefit of the Internet as a whole.

The conductor's role is essential in large-scale projects such as the Online Orchestra pilot performance, although had there been more time it would have been very interesting to explore the possibility of playing without a conductor. Indeed, there are further options to be explored here, such as having a conductor in each node (in, say, a project involving teachers and their classes); or indeed, perhaps, having an improvising soloist leading the performance - notes from the podium indeed. As Online Orchestra grows, it will be fascinating to see how the role of conductor evolves.

\section{References}

Byo, J. L. (1990), 'Recognition of intensity contrasts in the gestures of beginning conductors', Journal of Research in Music Education, 38:3, pp. 157-63.

Byo, J. L. and Austin, K. R. (1994), 'Comparison of expert and novice conductors: An approach to the analysis of nonverbal behaviors', Journal of Band Research, 30:1, pp. 11-25.

Lee, E., Wolf, M. and Borchers, J. (2005), 'Improving orchestra conducting systems in public spaces: Examining the temporal characteristics and conceptual models of conducting gestures', CHI05: Proceedings of the SIGCHI Conference on Human Factors in Computing Systems, Portland, Oregon, USA, 2-7 April, pp. 731-40. Miracle Theatre, (2014) 'Superfast tempest', http://www.miracletheatre.co.uk/2014/09/1752/. Accessed 23 December 2016. 
Prior, D., Reeder, P., Rofe, M., Biscoe, I. and Murray, S. (2017), 'Designing a system for Online Orchestra: Peripheral equipment', Journal of Music, Technology and Education, 10: 2-3, pp. 197-212.

Rofe, M. and Geelhoed, E. (2017), 'Composing for a latency-rich environment', Journal of Music, Technology and Education, 10: 2-3, pp. 231-56.

Rofe, M. and Reuben, F. (2017), 'Telematic performance and the challenge of latency', Journal of Music, Technology and Education, 10: 2-3, pp. 167-84.

Rofe, M., Geelhoed, E. and Hodsdon, L. (2017a), 'Experiencing Online Orchestra: Communities, connections and music-making through telematic performance', Journal of Music, Technology and Education, 10: 2-3, pp. 257-76.

Rofe, M., Murray, S. and Parker, W. (2017b), 'Online Orchestra: Connecting remote communities through music', Journal of Music, Technology and Education, 10: 23, pp. 147-66.

Rudolf, M. (1995), The Grammar of Conducting, 3rd ed., Boston, MA: Schirmer. Veronesi, D. (2014), 'Correction sequences and semiotic resources in ensemble music workshops: The case of conduction', Social Semiotics, 24:4, pp. 468-94.

Notes

1. In a recent telematic performance of The Tempest by Miracle Theatre (see www.miracletheatre.co.uk/2014/09/1752//), two actors played the part of Ariel, one per location. This enabled the character to appear to 'jump' between locations, at times appearing in real life, at times appearing telematically. 
Jonathan J. Hargreaves has asserted his right under the Copyright, Designs and Patents Act, 1988, to be identified as the author of this work in the format that was submitted to Intellect Ltd. 